\section{Human immunodeficiency virus infection and chemotherapy treatment in the Kingdom of Bahrain}

\section{Abstract}

Objective: This study investigated the officially registered and controlled HIV cases in Bahrain over the last 10-year period. Starting in 2011, a multidisciplinary team was established for HIV management involving major stakeholders; public health, infectious diseases, pharmacists, nursing and virologists.

Methods: A retrospective descriptive study is done about all HIV positive patients in Bahrain. Data was collected retrospectively from the 2014 registry for HIV patients, and their ART regimen, CD 4 count and viral load were obtained and entered in Excel sheet.

Results: A total of 208 patients were diagnosed to have HIV up to 2014. However, only 108 of them have all necessary data to be included in the study. In addition, a review of the frequency of admission of these patients over a 10-year period was performed. Review the medical records of the patients, indicated that the total of death among HIV positive patients were 38 (35.6\%) over the study period. Hepatitis $\mathrm{C}$ was the most common co-infection observed among 26 patients (24\%), followed by tuberculosis with 24 patients (22.2\%).

Conclusion: It was found that usage of ART treatment regimen of efavirenz, darunavir and lopinavir, better structure of HIV team and program, had helped to control better the patients, and there was a clear increase in the number of patients with improving CD4 count. Based on the results of this study, the HIV multi-disciplinary management team is found an essential part for the best management of these patients.
Jameela Al Salman', Rawan Al Agha', Eman Fareed', Leena Ali', Kubra Nasser, Zainab AlSikri', Rehab Al Noaimi

1 Salmaniya Medical Complex, Manama/Bahrain.

Corresponding author:

\section{Dr. Jameela Al Salman}

$\equiv$ jsalman@health.gov.bh, jalsalman@hotmail.com 
Key Words: Infection, HIV, Antiretroviral therapy

\section{Introduction}

Human immunodeficiency virus (HIV) is a lentivirus which is a subgroup of retrovirus that causes acquired immunodeficiency syndrome (AIDS) and predispose for opportunistic infections and malignancies. HIV infects certain cells in the immune system such as helper T cells (CD 4 T cells in particular), macrophages and dendritic cells. Many of the individuals who are HIV-positive can be unaware of their infection [1]. According to global studies in 2012, it is estimated that 35.5 million people were living with the virus. Of these, 2.1 million were adolescents aged between 10-19 years, of which the girls accounted to $56 \%$ [2]. The majority of HIV infections in the sub-Saharan Africa were adolescents . Outside Africa, South Asia had the highest number of HIV positive adolescents, estimated to be $6 \%$ of the global burden. These were followed by East Asia and Pacific (110.000), Latin America and the Caribbean (81.000), Eastern Europe and Central Asia $(22,000)$ and the middle East and North Africa $(17,000)$ [3].

In 2013, 1.5 million people died of AIDS, a 35\% decrease since 2005. The number of deaths has decreased in part due to available of antiretroviral treatment (ART). Multiple prevention strategies exist to combat the virus including recommending circumcision, use of condoms, behavior changes, and blood supple safety. On the other hand, HIV treatment includes both ART and other medication to prevent and treat opportunistic infections. Globally, it is estimated that $37 \%$ of people living with HIV are getting treatment and around $75 \%$ of all people receiving antiretroviral therapy live in sub-Saharan Africa [4].The publication and the availability of data about the epidemiology of HIV in the middle-east region is very limited. Our research provides some of these data about the kingdom of Bahrain.

In the Kingdom of Bahrain, screening for HIV sta-
Table 1. Demographic characteristics of HIV examined patients

\begin{tabular}{|l|c|}
\hline \multicolumn{1}{|c|}{ Items* } & No.(\%) \\
\hline Total no. of registered patients & $108(100)$ \\
\hline $\begin{array}{l}\text { Total no. of admitted patients to regular } \\
\text { treatment }\end{array}$ & $95(87.9)$ \\
\hline Males & $73(67.6)$ \\
\hline Females & $22(20.4)$ \\
\hline Recurrent 3 admissions & $23(21.5)$ \\
\hline The average age of admitted cases & $37-y e a r$ \\
\hline CD4 count less than200ug/dl & $23(21.3)$ \\
\hline CD4 count between200-500ug/dl & $31(28.7)$ \\
\hline CD4 count more than & $38(35.19)$ \\
\hline
\end{tabular}

*38/76 (50\%) had detectable viral load

tus started in 1989. The HIV positive patients have a registry in the public health sector of the Ministry of Health. The patients get a regular medical follow-up for their health status via outpatient clinic appointments. A retrospective descriptive study is done about those patients, their current treatment regimens and other blood parameters aiming to have a general idea about their health status in order to help in their medical care.

\section{Methods and Materials}

In Bahrain, a multidisciplinary team was established in 2011, for HIV management involving major stakeholders: public health, infectious diseases, pharmacists, nursing and virologists. The team established a system which capture each step of patients care to improve its quality. This team meets regularly, puta a complete action plan to form an integrated system to provide the best care for these patients.

We started collecting the basic data which involved the followings:

First form: The demographic information about the patients, their risk factors, their progress and 
response to treatment and their follow up, their prevention protocol.

The second form include the serial of their blood tests specifically, HIV viral load, CD4 count and the genotyping.

Third form: Lists from the pharmacy where we assigned one pharmacist to look after these patients, where we get an updated list of the patients with their medications, and if they missed taking them to call them urgently to ensure compliance.

We assigned a nurse coordinator for the HIV service who overlooks the whole process of data collection, entering and providing patient support services

These data were entered in a soft copy excel sheets to analysis the data. Over the same period we introduced new and updated HIV medications to increase the response rate to HIV medications.

A software database was established to include all patients: to record their visits, labs results, clinical progress, medications regimen and their appointments to flag missed ones.

The data was collected retrospectively from the current created registry about all patients who are currently living with HIV and are followed in the same hospital. This hospital is a secondary and tertiary care hospital and the only hospital in the kingdom of Bahrain that provide care for HIV patients. Their ART regimen, CD 4 count and viral load were gathered and entered in Excel sheet. In addition, a review of the frequency and reason of admission, the most common infections and the mortality rate for all cases of HIV that were admitted to the same hospital over a 10 year period. The information was collected during the period 2004 to 2014 from the medical records of patients. All data including their names were dealt with in complete privacy. All patients were included without any exclusion criteria.

\section{Results}

A total of 208 patients were diagnosed to have HIV and many of them are still alive up to 2014. However, only 108 of them have their full data (including house address and contact details) documented. The rest of them have no trace. Of those 108 patients with full data and regular clinic follow up, only 95 are on regular treatment. The other 13 patients are not attending their outpatient HIV clinic appointments. Out of the 95 patients, 73 are males and 22 are females.

In addition, the medical records of all HIV patients who were admitted over the last ten years from 2004 till 2014, it was found that the total admission of HIV positive patients were 108. Of those, 23 had recurrent admissions with an average number of three admissions per patient. The average age of admitted cases was 37 years.

Hepatitis C was the most common co-infection among those patients and accounted to $24 \%$. It was followed by Hepatitis B with $3.73 \%$, and only $3 \%$ had double infection with both types of viruses (B and $C$ ).

The CD4 count was done to the patients upon diagnosis of the virus which varied in year from one to another and repeated thereafter as mandated by the patients' condition. According to the first CD4 count it showed that 26 out of the 95 patients had a count of less than 200ug/dl, 36 had count between 200-500ug/dl and 30 were more than 500ug/dl. Three patients had no CD4 counts due to laboratory error [Figure 3]. The repeated CD4 count showed 23 with count less than 200ug/ dl, 31 with count between 200-500ug/dl and 38 with CD4 more than $500 u g / d l$. For one patient it was not repeated since diagnosis and two other the sample was not processed by the laboratory due to uncertain error [Table 2]. The CD4 count was repeated on average two to three years after the patients' first collected sample and initiating his treatment.

The viral load was done to 76 of the patients on treatment and it showed that 38/76 (50\%) had 
Table 2. Treatment regimen of the 95 HIV patients

\begin{tabular}{|l|l|}
\hline \multicolumn{1}{|c|}{ Drug combination } & No. (\%) \\
\hline Efavirenz based regimen & $49(51.6)$ \\
\hline Darunavir based regimen & $31(32.6)$ \\
\hline Lopinavir based regimen & $15(15.7)$ \\
\hline
\end{tabular}

detectable viral load, target not detected in 38/76 (50\%) from the total patients who had viral load done and $40 \%$ form the total number of patients who are currently on treatment.

A total of 95 patients were on different medication regimens. Of these, 49(51.6\%) were on efavirenz based regimen and $31(32.6 \%)(n=31)$ were on darunavir based regimen and 15 on lopinavir based regimen (15.7\%) as shown in Table 3.

Thirteen out of the 107 patients presented with respiratory symptoms such as shortness of breath and cough or vague symptoms like fever and were admitted and managed as pneumonia without isolating a specific organism. On the other hand, 21 patients were diagnosed to have respiratory Tuberculosis (TB), three with military TB and two with recurrent TB due to drug non-compliance upon discharge at the first admission. One case had TB lymphadenitis [Table 2]. Their diagnosis was confirmed using AFB of sputum and bronchial wash or PCR for detection AFA, in addition to the clinical picture. Twelve patients were admitted with suspected sepsis without proving the agent of infection. Others had various reasons for admission such as drug overdose, myocardial infarction, alcohol intoxication and gastrointestinal bleed. Regarding central nervous system infections, one diagnosed with viral encephalitis, three cases with cryptococcal meningitis and two patients with toxoplasma encephalitis. The mode of presentation varied. Nonetheless, oral candidasis was a common coincidental finding in many patients. One patient was diagnosed with Kaposi sarcoma after presenting with violacous skin lesions where a biopsy was taken.
Table 3. Major microbial infections observed among 108 HIV Patients

\begin{tabular}{|l|c|}
\multicolumn{1}{c|}{ Microbial infections } & No. (\%) \\
\hline Hepatitis C & $26(24)$ \\
\hline Hepatitis B & $4(3.7)$ \\
\hline Hepatitis C \& Hepatitis B & $3(3)$ \\
\hline Pulmonary tuberculosis & $21(19.6)$ \\
\hline Extra-pulmonary tuberculosois & $4(3.7)$ \\
\hline Cryptococcal meningitis & $3(2.8)$ \\
\hline Toxoplasma encephalitis & $2(1.9)$ \\
\hline Kaposi sarcoma & $1(0.9)$ \\
\hline Candidasis & Null
\end{tabular}

Fourteen patients had positive deep tracheal aspirate(DTA) / sputum. The organisms grown were various such as $S$ aureus, Pseudomonas spp., and E.coli and multi-drug resistant Acintobacter spp. Some DTA cultures have grown multiple organisms [Table 4] .

\section{Discussion}

There has been significant scale-up of the use of

Table 4. Profile of bacteria isolates from sputum / DTA and associated with respiratory tract infections among HIV patients

\begin{tabular}{|c|c|c|}
\hline Organism Type* & Sensitivity & No. \\
\hline Staph aureus & Highly susceptible** & 2 \\
\hline E.coli (ESBL) & Susceptible only to Carbapenems & 3 \\
\hline $\begin{array}{l}\text { Methicillin } \\
\text { resistant S. aureus }\end{array}$ & Susceptible only to Vancomycin & 1 \\
\hline E. coli & Highly susceptible & 1 \\
\hline $\begin{array}{l}\text { Pseudomonas } \\
\text { spp. }\end{array}$ & Highly susceptible & 2 \\
\hline $\begin{array}{l}\text { Acintobacter spp. } \\
\text { (MDR) }\end{array}$ & Susceptible to colistin/ tegicycline & 3 \\
\hline $\begin{array}{l}\text { Klebsiella spp. } \\
\text { (ESBL-producer) }\end{array}$ & Susceptible only to Carbapenems & 2 \\
\hline Klebsiella spp. & Highly susceptible & 3 \\
\hline
\end{tabular}

* Some culture specimens grew more than one bacteria spp.

**To most tested antibiotics 
ART especially with the increase incidence of HIV positive patients. However, drug compliance is the cornerstone in the successful of HIV infection management. The success of any treatment plan relies on life-long, high degree of medication adherence to have the best clinical outcome and reduce the incidence of drug resistance. Reviews of over 50 years of research studies have shown that drug compliance rates above $80 \%$ for treating various chronic illnesses has been a serious issue even in well developed rich countries. Therefore achieving good rates of medication compliance in under developed poor resource countries is a big challenge and must be closely monitored [5]. Our review showed a CD4 count response among the patient over the years which indicate good drug compliance by them. It is highly important for HIV patients to be admitted for continuous clinic visit, regular laboratory tests and medication adherence [6].

According to recent reports from Middle East and Middle East region (MENA), $60 \%$ of HIV infections among women occur before the age of 20 and the more than $50 \%$ of the new infection are seen in the 15-24 age groups [7]. It was found that of the nearly 500,000 infected individuals with HIV in the region; almost half of them are females. In addition, sexual transmission of HIV is increasing in the region. Married women in particular are among the most vulnerable to HIV infection due to the fact that they do not see themselves as being so because of social structure and customs that surrounds them [8]. Actually these customs do not only surround women but it prevails men too. For example, young men are assumed not to be sexually active until they are married and married men are faithful to their wives with no extra-marital relations. However, the prevalence of sexually transmitted disease is fairly high in some regions according to certain studies that indicate much extra-marital unprotected sexual relations [9].Therefore, thorough history from all patients despite their background is advised for early detection of new cases which will influence their diagnosis, management and transmission to other partners.

Proper understanding of the accurate HIV epidemic in MENA region has been sluggish due to problems inherent to low HIV prevalence in the population compared to other population. In addition, the pace of the development of surveillance systems is slow with insufficient data collection and analysis[10]. Despite the number of people living with HIV in the MENA countries has increased over the last decade, the current prevalence of $0.1 \%$ is still among the lowest rates worldwide. Five countries including Algeria, Islamic Republic of Iran, Morocco, Somalia, and Sudan accounted for $88 \%$ of these people living with HIV infection [11].

The stigma and discrimination behind the people living with HIV is a major factor in the MENA region that creates difficulty in approaching hidden population with positive HIV. The moral views towards the HIV positive people in most Arab/ Muslim countries both in the community and the health system is a major obstacle for patients to seek care and treatment. In a study done in Egypt included 153 HIV positive patients showed $51.6 \%$ feeling stigmatized by others in the community and $43.1 \%$ reported changes in people's views towards them. In addition, $66.7 \%$ isolated themselves from the community because fear of disgrace, $44.4 \%$ noticed changes in the behaviors of their relatives and $53.6 \%$ felt useless to their community [12].

Based on our study results, we found that with better ART treatment and better structure of HIV team and program, we are getting more patients to be controlled. There is a clear increase in the number of patients with improving CD4 count. There is also a higher number of patients with undetectable viral load. Reports showed that ART lowers the HIV transmission possibility and as the ART coverage rate increases, HIV incidence decreases. ART is recommended in all HIV positive cases despite their CD 4 count which is termed "Treatment as prevention" The rate of ART coverage has increased in the re- 
gion during the last decade. However, by the end of 2013, the ART coverage in the region was still lower compared to countries in other regions throughout the world. The coverage was estimated to be around only $11 \%$. Despite significant efforts made by all countries in the region to increase the number of people receiving treatment, the fast growing number of new HIV cases is a major obstacle to close the gap. In 2013, less than one out of eight positive patients living with the virus eligible for ART received it in Djibouti, Iran, Egypt, Somalia, Sudan, and Yemen. As a result, the region has experienced a significant increase in mortality due to AIDS which has reached $17 \%$ in 2012. [13]

The high rate of hepatitis C co-infection is because that the majority of HIV patients are IV drug users. Drug addiction is a persistent and growing problem in MENA. According to the United Nations Office on Drugs and Crime, $83 \%$ of the world supply of heroin is produced in Afghanistan, and $>75 \%$ of this is marketed through Iran. The number of injecting drug abusers in MENA is estimated to be 335, 000-1, 635 000. Drug abuse was the most common mode of HIV transmission among men in Iran (73.5\%) and Afghanistan (60.2\%) in 2011. While Iran has had the highest numbers of positive HIV patients among all countries in the region (185 000), it is followed by Pakistan (117 000) and Egypt (89 000), where injecting drug use contributed to $20 \%$ and $23 \%$, respectively, of all newly notified cases in 2011; and the lowest number (1000) was reported in Somalia. [14]

Opportunistic infections are commonly observed in HIV patients who have limited medical care or a consequence of medication with non compliance / virus resistance to ART. A retrospective study conducted in Bahrain between the period of 2009-2013 that aimed to examine the prevalence of opportunistic infections and its relation to absolute CD4 count (CD4 \% and CD4/CD8) ratio. The results showed CD4\% and absolute CD4 count in HIV patients with associated infection was significantly lower than in those without evidence of opportunistic infections (157 \pm 295 and $471 \pm 285$, respectively) with $p<$ 0.001 . The most common opportunistic infections AIDS patients were due to Staphylococcus aureus followed by yeast infections $(28.7 \%$ and $27.2 \%$, respectively). PCP was present in 15.1\%, Mycobacterium Tuberculosis and Mycobacterium other than tuberculosis in $10.6 \%$ and $7.5 \%$ respectively in opportunistic infections group patients [15]. Our report roughly investigated the frequency of infections in HIV and AIDS group patients without segregation. The majority had symptoms and signs indicating an infection, however, many of them were treated empirically without isolating the causative organism. This could be due to defect in collecting the proper samples or delay in the collection specimens for septic work-up. For those who have a significant isolated organism, Mycobacterium tuberculosis was the leading cause of infection either in respiratory tract or extra-pulmonary site.

\section{Conclusion}

Based on the results of this study, the HIV multidisciplinary management team is an essential part for the best management of HIV patients. This approach will also decreases developing opportunistic infections. Education about the disease should be encouraged in the society. Equally important is surveillance among the high risk groups (sex workers and drug abusers) for early detection of infected individuals. The social stigmata of having the virus are a main concern among those patients which prohibits many of them to approach medical care and receive the proper treatment.

\section{References}

1. Dodd RY, Notari EP 4th, Stramer SL. Current prevalence and incidence of infectious disease markers and estimated window- 
period risk in the American Red Cross blood donor population. Transfusion 2002; 42:975.

2. United Nations Children's Fund (UNICEF). UNICEF Analysis of UNAIDS2012 HIV and AIDS Estimates. New York, NY: UNICEF; 2013.

3. Joint United Nations Programme on HIVIAIDS (UNAIDS). Global AIDS.Response Progress Reporting 2014: Construction of Core Indicators for.Monitoring the 2011 UN Political Declaration on HIV and AIDS.Geneva, Switzerland: UNAIDS; 2014.

4. WHO/UNAIDS/UNICEF. Global update on HIV treatment 2013; June 2013.

5. DiMatteo MR. Variations in patients' adherence to medical recommendations. Med Care. 2004;42:200-209.

6. Liu H, Golin CE, Miller LG, Hays RD, Beck CK, Sanandaji S, Christian J, Maldonado T, Kaplan AH, Wenger NS. A comparison study of multiple measures of adherence to HIV protease inhibitors. Ann Intern Med. 2001;134: 968-977.

7. UNAIDS. [May 24, 2008];Report on the Global Epidemic. 2006 Available at: http://www.unaids.org/.

8. Global Network of Researchers on HIVIAIDS in the Middle East and North Africa. Investing in research and education: GNR/ MENA defines the struggle against HIVIAIDS in the Middle East and North Africa. [June 10, 2008]; 2003 Available at: http:// www.uic.edu/orgs/gnr-mena/gnr-mena\%20booklet.

9. Obermeyer Carla Makhlouf. HIV in the Middle East. BMJ. 2006; 333:21.

10. Abu-Raddad LJ, Ghanem KG, Feizzadeh A, Setayesh H, Garcia Calleja JM, Riedner G.HIV and other sexually transmitted infection research in the Middle East and North Africa: promising progress? Sex Transm Infect 2013;89:iii1-4.

11. Bozicevic I, Reidner G, Haghdoost A. HIV case reporting in the countries of North Africa and the Middle East. J Int AIDS Soc 2014;17:18962.

12. Kabbash IA, El-Gueneidy M, Sharaf AY, Hassan NM, Al-Nawawy AN. Needs assessment and coping strategies of persons infected with HIV in Egypt. East Mediterr Health J 2008;14:1308-20.

13. UNAIDS report on the global AIDS epidemic 2013. UNAIDS; 2013. Available at: http://www.unaids.org/sites/default/files/ media_asset/UNAIDS_Global_Report_2013_e_1. (Published August 8, 2015).

14. Mumtaz GR, Weiss HA, Thomas SL, et al. HIV among people who inject drugs in the Middle East and North Africa: systematic review and data synthesis. PLoSMed 2014;11:e1001663.

15. Nermin K Saeed, Eman Farid, Afaf E Jamsheer. Prevalence of opportunistic infections in HIV-positive patients in Bahrain: a four-year review (2009-2013). J Infect Dev Ctries 2015; 9(1):060-

\section{9. doi:10.3855/jidc.4997.}

\section{Comment on this article:}
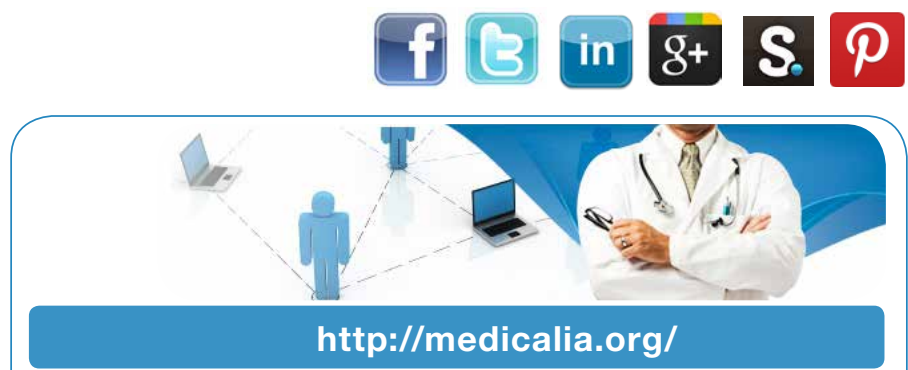

Where Doctors exchange clinical experiences, review their cases and share clinical knowledge. You can also access lots of medical publications for free. Join Now!

\section{Publish with iMedPub}

\section{http://www.imed.pub}

The Journal is an open access peer-reviewed journal that publishes scientific papers about all aspects of antimicrobials. The journal will publish original research articles, reviews, brief reports and case reports dealing with basic and clinical antibacterial agents, antiviral, antiprotozoals, antituberculuous, antifungal and antihelminthes agents.

All manuscripts must be prepared in English, and are subject to a rigorous and fair peer-review process. Accepted papers will immediately appear online.

The journal aims to advance the knowledge, attitude and the research of chemotherapy in the Arabic world in cooperation with international, national scientific and public societies as well as research centers with similar aims and objectives. 